\title{
ACM TIST Special Issue on Data-Driven Intelligence for Wireless Networking
}

Data has become a key asset in today's wireless network design. Protocols, strategies, and applications/services in wireless networks can benefit from the intelligence mined and learnt from data collected from the widely deployed "sensors" in the network. Carrying out analysis of data patterns and trends to make intelligent decisions for today's wireless network design is appealing to both academia and industry. The aim of this special issue is to promote the state of the art in scientific and practical research on big data intelligence for wireless network design and to bring together researchers and practitioners from academia and industry in an effort to present their research work and share research and development ideas.

The articles in this special issue span a wide range of wireless network measurement and deployment, data-driven wireless applications, and privacy and security.

\section{Wireless Deployment and Measurement}

Two articles focus on wireless deployment and measurement.

On wireless network deployment, the article entitled "DMAD: Data-Driven Measuring of Wi-Fi Access Point Deployment in Urban Spaces” studies wireless network deployments that can achieve multiple objectives, such as traffic offloading, network coverage, and indoor localization. Shen et al. propose measurements of a Wi-Fi access point deployment. These measurements, combined with shop profiles from the Internet, not only estimate potential dead spots of the AP deployment but also quantify their severity.

The article entitled "Exploring Indoor White Spaces in Metropolises" exploits white spaces to meet the rapidly growing demand for wireless data services in both outdoor and indoor scenarios. Chen et al. conduct a large-scale measurement study and compare outdoor and indoor TV spectrum occupancy at $30+$ diverse locations in a typical metropolis. They show that abundant white spaces are available in different areas. They propose a data-driven, low-cost indoor white space identification system for White-space Indoor Spectrum EnhanceR (WISER) to allow secondary users to identify white spaces for communication without sensing the spectrum themselves.

\section{Data-driven Applications}

Five articles use wireless data intelligence for new applications.

"Energy Efficient Mobile Video Streaming: A Location-aware Approach" studies the problem of energy efficient mobile video streaming, makes use of the observed correlation between bandwidth and user location, and also observes that a user's location is predictable in many situations. Zhang et al. propose an optimal offline algorithm to achieve high-quality video playback while minimizing energy use. The proposal learns from historical location-aware bandwidth conditions and predicts future bandwidths along a planned route to make online wireless download decisions.

In "TensorBeat: Tensor Decomposition for Monitoring Multi-Person Breathing Beats with Commodity WiFi," Mao et al. propose leveraging the tensor decomposition technique to estimate the breathing rates of multiple persons with commodity WiFi devices by using the channel state information (CSI) phase difference data. The authors develop a stable signal-matching algorithm that achieves high accuracy under different environments.

(c) 2017 ACM 2157-6904/2017/09-ART4 \$15.00

https://dx.doi.org/10.1145/3104984

ACM Transactions on Intelligent Systems and Technology, Vol. 9, No. 1, Article 4. Publication date: September 2017. 
Radio Frequency Identification (RFID) applications involve sensing and recognizing tag mobility. Existing RFID localization methods, however, are mostly designed for static or slowly moving targets. The article entitled "i2tag: RFID Mobility and Activity Identification through Intelligent Profiling" presents an intelligent mobility-aware activity identification system for RFID tags in multipath-rich environments that can quantify different levels of mobility. The proposal employs a supervised learning framework based on fine-grained mobility profiles, using features that include RSSI variance, packet loss rate, and a novel relative-phase-based fingerprint.

"UMCR: User Interaction-Driven Mobile Content Retrieval" studies content retrieval for mobile applications. Wang et al. propose and implement a user interaction-driven mobile content retrieval (UMCR) system that combines the user interaction path traversing (UIPT) and Deep Packet Inspection (DPI) together to obtain mobile content. UIPT determines the events of user interactions in various applications to capture the static content such as text and images, in which a traversal depth termination scheme and an optional cut-off component are adopted to balance the content coverage and traversing efficiency. Meanwhile, the analysis based on DPI is responsible for extracting the videos as well as extracting the infrastructure information and performance metrics.

The article entitled "Taking the Pulse of U.S. College Campuses with Location-Based Anonymous Mobile Apps" uses the data-driven approach enabled by today's mobile apps to study campuses. Keith et al. show that students in all regions (northeast, south, midwest, and west) yakked about academics at rates between $7 \%$ and $9 \%$, with no statistically significant differences between regions, and that campuses with higher rates of yakking about dating and sex had lower rates of academic-related yaks.

\section{Privacy and security}

Two articles address privacy of people using wireless devices.

The article entitled "From Electromyogram to Password: Exploring the Privacy Impact of Wearables in Augmented Reality" studies the potential privacy leakages from always-on wearable devices. Zhang et al. demonstrate that the coarse access control on today's AR system could lead to possible abuse of sensor data. They show that sensitive information, such as passwords typed on a keyboard and PIN sequence entered through a touchscreen, can be recovered with a mean success rate of $91 \%$.

On privacy of IoT, the article entitled "Secure IoT-based, Incentive-aware Emergency Personnel Dispatching Scheme with Weighted Fine-grained Access Control" show that effective emergency response plays an important role in a successful rescue and proposes a secure IoT-based and incentive-aware emergency personnel dispatching scheme with weighted fine-grained access control.

By no means can one special issue scope all the exciting research in the field of data-driven design for intelligent wireless networking. In addition, more challenges are emerging with more real-time data sensing and processing capacities and more complicated wireless network infrastructure. We thank all the authors for their contributions and hope these articles can stimulate further research. We appreciate the valuable inputs from our reviewers, and we are grateful to the editorial board of ACM TIST for their help in coordinating the publication process. We hope you will enjoy reading this special issue.

Wenwu Zhu
Jean Walrand
Yike Guo
Zhi Wang
Guest Editors 